\title{
Long-term follow-up of mandibular dental arch changes in patients with complete non-syndromic unilateral cleft lip, alveolus, and palate
}

Sariesendy Sumardi ${ }^{1}$, Benny S. Latief ${ }^{2}$, Anne Marie Kuijpers-Jagtman ${ }^{\text {Corresp., 3, 4, } 5}$, Edwin M. Ongkosuwito ${ }^{6,7}$, Ewald M. Bronkhorst ${ }^{7}$, Mette A.R. Kuijpers ${ }^{6,7}$

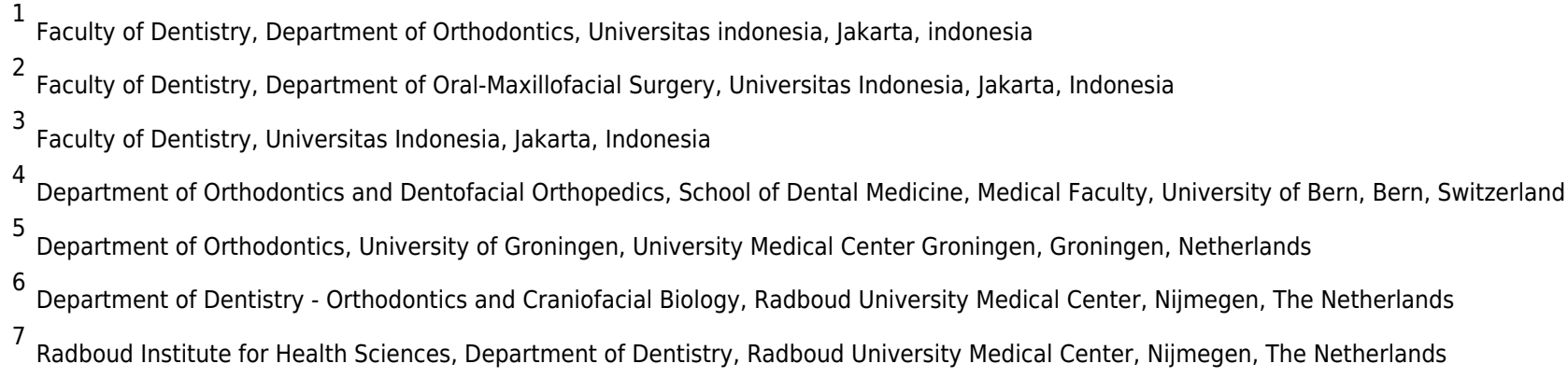

Background. Treatment of cleft lip and palate (CLP) requires a comprehensive interdisciplinary approach and long-term follow-up. Only a few studies are available that reported on changes after treatment, which showed that in particular the transverse dimension, in patients with CLP is prone to changes after treatment. However, those studies did not pay attention to concomitant changes in the mandibular arch that occur after treatment. Objectives. To evaluate mandibular transverse dental arch dimensions and interarch transverse changes in patients with complete non-syndromic unilateral cleft lip, alveolus, and palate (CUCLAP) up to five years after treatment. Material and methods. Retrospective longitudinal study in 75 consecutive patients with CUCLAP (197 mandibular dental casts) directly after comprehensive treatment (T0), two (T2), and 5 years after treatment (T5). Great Ormond Street, London and Oslo (GOSLON) scores were available for all patients. Three-dimensional scans of all dental casts were made. Inter premolar and intermolar distances between the mandibular contralateral teeth were measured. The modified Huddart Bodenham (MHB) was applied to assess the transverse interarch relationship. Paired t-tests and ANOVA were used to analyze transverse and interarch transverse changes. Linear regression analysis was done to define contributing factors. Results. Paired t-tests showed a significant decrease of the mandibular inter first and second premolar distances $(p<0.05)$ and an increase of the inter second molar distance, whilst the MHB Index deteriorated at all time points for all segments and for the total arch score $(p<0.05)$. Linear regression showed no significant contributing factors on 
the decrease of the transverse distances. However, inter arch transverse relationship was significantly affected by age at the end of treatment, missing maxillary lateral incisor space closure, and the GOSLON Yardstick score at the end of treatment $(p<0.05)$, especially during the first two years after treatment. Conclusions. Changes occurred in the mandibular arch expressed as changes in the transverse dimensions and interarch relationship measured by the MHB Index. A younger age at the end of treatment, space closure for a missing maxillary lateral incisor and a higher GOSLON score at the end of treatment negatively influence the interarch transverse deterioration especially in the first two years after treatment. For the transverse dimensional changes in the mandibular arch such influencing factors could not be determined. 


\section{Long-term Follow-up of Mandibular Dental Arch}

3 Changes in Patients with Complete Non-syndromic

4 Unilateral Cleft Lip, Alveolus, and Palate

5

6

7

\section{8} 9

Sariesendy Sumardi ${ }^{1}$, Benny S. Latief ${ }^{2}$, Anne Marie Kuijpers-Jagtman ${ }^{3,4,5}$, Edwin M.

Ongkosuwito $^{6,7}$, Ewald M. Bronkhorst ${ }^{7}$, Mette A. R. Kuijpers ${ }^{6,7}$

${ }^{1}$ Department of Orthodontics, Faculty of Dentistry, Universitas Indonesia, Jakarta, Indonesia

${ }^{2}$ Department of Oral-maxillofacial Surgery, Faculty of Dentistry, Universitas Indonesia, Jakarta, Indonesia

${ }^{3}$ Department of Orthodontics and Dentofacial Orthopedics, School of Dental Medicine, Medical Faculty, University of Bern, Bern, Switzerland

${ }^{4}$ Faculty of Dentistry, Universitas Indonesia, Jakarta, Indonesia

${ }^{5}$ Department of Orthodontics, University Medical Center Groningen, University of Groningen, Groningen, The Netherlands

${ }^{6}$ Department of Dentistry - Orthodontics and Craniofacial Biology, Radboud University Medical Center, Nijmegen, The Netherlands

${ }^{7}$ Radboud Institute for Health Sciences, Department of Dentistry, Radboud University Medical Center, Nijmegen, The Netherlands

Corresponding Author:

Anne Marie Kuijpers-Jagtman, DDS PhD, FDSRCS ${ }^{4}$

Universitas Indonesia, Faculty of Dentistry

Salemba Raya 4, Jakarta Pusat, Jakarta, 10430, Indonesia

Email address: a.m.kuijpers-jagtman@umcg.nl 


\section{Abstract}

31 Background. Treatment of cleft lip and palate (CLP) requires a comprehensive interdisciplinary

32 approach and long-term follow-up. Only a few studies are available that reported on changes

33 after treatment, which showed that in particular the transverse dimension, in patients with CLP is

34 prone to changes after treatment. However, those studies did not pay attention to concomitant

35 changes in the mandibular arch that occur after treatment.

36 Objectives. To evaluate mandibular transverse dental arch dimensions and interarch transverse

37 changes in patients with complete non-syndromic unilateral cleft lip, alveolus, and palate

38 (CUCLAP) up to five years after treatment.

39 Material and methods. Retrospective longitudinal study in 75 consecutive patients with

40 CUCLAP directly after comprehensive treatment (T0), two (T2), and 5 years after treatment

41 (T5). Great Ormond Street, London and Oslo (GOSLON) scores were available for all patients.

42 Three-dimensional scans of all dental casts were made. Inter premolar and intermolar distances

43 between the mandibular contralateral teeth were measured. The modified Huddart Bodenham

44 (MHB) was applied to assess the transverse interarch relationship. Paired t-tests and ANOVA were used to analyze transverse and interarch transverse changes. Linear regression analysis was done to define contributing factors.

Results. Paired t-tests showed a significant decrease of the mandibular inter first and second premolar distances $(\mathrm{p}<0.05)$ and an increase of the inter second molar distance, whilst the MHB Index deteriorated at all time points for all segments and for the total arch score $(p<0.05)$. Linear regression showed no significant contributing factors on the decrease of the transverse distances. However, inter arch transverse relationship was significantly affected by age at the end of treatment, missing maxillary lateral incisor space closure, and the GOSLON Yardstick score at the end of treatment $(p<0.05)$, especially during the first two years after treatment. dimensions and interarch relationship measured by the MHB Index. A younger age at the end of treatment, space closure for a missing maxillary lateral incisor and a higher GOSLON score at the end of treatment negatively influence the interarch transverse deterioration especially in the first two years after treatment. For the transverse dimensional changes in the mandibular arch such influencing factors could not be determined.

60 Keywords: treatment outcome, cleft palate, dental arch, mandible, maxilla, orthodontics 


\section{Introduction}

64 Unilateral cleft lip and palate is one of the most common birth defects. Most unilateral cleft lip,

65

66

67

68

69

70

71

72

73

74

75

76

77

78

79

80

81

82

83

84

85

86

87

88

89

90

91

92

93

94

95

96

97

98

99

100

101

102 alveolus, and palate (UCLAP) are non-syndromic (Dixon et al., 2011) with a prevalence of 1.37 per 1,000 births in low- and middle-income countries (Kadir et al., 2017). Mossey and Modell (2012) estimated the prevalence per 1,000 births in South East Asia being1.08, Middle East 0.72, North America 1.17 and in Europe ranging from $0.63-1.07$. Also, it has been found that the prevalence of cleft lip and palate (CLP) in males is higher than in females (Martelli et al., 2012; Y1lmaz, Özbilen \& Üstün, 2019).

It has already been recognized for a long time that treatment of these birth defects requires a comprehensive interdisciplinary approach. The American Cleft Palate-Craniofacial Association (2018) developed guidelines for team treatment for patients with orofacial clefts. Whilst the team provides comprehensive and integrated treatment, follow-up of the patients is also an integral part of the team duties. Following patients over a long period of time is not only important to assess treatment outcome within a team, but also to compare the results with other centers, in order to improve the care of patients with clefts.

Maxillary dental arch transverse dimensions and dental arch relationships following different treatment protocols for patients with orofacial clefts have been the topic of studies and systematic reviews that evaluated treatment outcome in relation to different treatment parameters such as timing of palatal surgery in relation to craniofacial outcome (Kappen et al., 2018), timing of primary alveolar bone grafting (Carbullido et al., 2021), outcome of maxillary distraction (Kloukos et al., 2018), and rehabilitation of the maxillary arch with a dental implant or an anterior fixed partial denture replacing the missing lateral incisor at the cleft side (Caballero et al., 2019; Rezende Pucciarelli et al., 2020, 2021; Soares et al., 2021a,b). However, those studies did not pay much attention to concomitant changes in the mandibular arch of patients with cleft lip and palate that occur after treatment. To our knowledge no studies are available in the literature that describe transverse changes in the mandibular arch. It is well-known that the maxillary arch, and in particular the transverse dimension, in patients with a cleft palate is prone to changes after treatment (Marcusson and Paulin, 2004; Al-Gunaid et al., 2008; Semb, Rønning \& Åbyholm, 2011; Rezende Pucciarelli et al., 2020; Soares et al., 2021a,b). Especially because often a considerable amount of expansion is needed during the orthodontic treatment to overcome the constrictive effect of palatal scar tissue. It may be possible that the mandibular arch will adapt, at least - partly, to post-treatment changes in the maxillary arch, but it may also be possible that the mandibular arch remains stable. This would result in disturbance of the transverse relationship between the arches.

Several factors could influence mandibular arch changes and interarch transverse relationship after treatment, such as sex and age at the end of treatment, orthodontic opening or closing of the space of a missing maxillary lateral incisor (Oosterkamp et al., 2010), extractions in the mandibular arch, expansion of the maxillary arch in relation to secondary bone grafting (Emodi et al., 2015), and interarch relationship as represented by the GOSLON (Great Ormond Street, London and Oslo) Yardstick at the end of treatment. These factors may predispose to 
103

104

105

106

107

108

109

110

111

112

113

114

115

116

117

118

119

120

121

122

123

124

125

126

127

128

129

130

131

132

133

134

135

136

137

138

139

140

141

142

changes in the dental arches for which the operator should anticipate with the orthodontic retention appliances.

The aim of this study was to determine long term changes in transverse mandibular arch dimensions and to determine interarch transverse relationship in patients with a complete nonsyndromic unilateral cleft lip, alveolus, and palate (CUCLAP) until 5 years post-treatment. The null hypothesis was transverse mandibular arch dimensions and the transverse interarch relationship in patients with CUCLAP do not change until 5 years post orthodontic treatment. The risk factors that could affect the stability of the transverse mandibular arch dimensions and the transverse interarch relationship were also studied.

\section{Materials \& Methods}

\section{Subjects and treatment protocol}

This is a retrospective longitudinal study in patients with a non-syndromic CUCLAP. Inclusion criteria were: non-syndromic as ascertained by the clinical geneticist, Caucasian, born between 1 January 1983 - 31 December 1992, Simonart's band allowed, consecutively treated from birth on with the same treatment protocol in the Cleft Lip and Palate Craniofacial Center, Radboud University Medical Center, Nijmegen, The Netherlands. Exclusion criteria were: additional craniofacial anomaly present, incomplete cleft, incomplete or submucous cleft lip at the other side, not Caucasian, primary operations performed elsewhere, treatment not yet finished.

All surgical procedures were performed by two experienced cleft surgeons and all patients were treated according to a standardized treatment protocol. The treatment protocol consisted of infant orthopedics followed by lip repair (Millard) at 4-8 months, soft palate repair (modified von Langenbeck) at 12-14 months, superiorly based pharyngeal flap at 4-5 years (if needed), maxillary protraction and expansion at 5-6 years for 12-18 months (if needed) followed by minor lip/ala nose correction (if needed), alignment of maxillary incisors and/or maxillary expansion at 9-11 years with a quad-helix or removable appliance followed by secondary bone grafting with a chin or rib graft together with hard palate closure (Boyne \& Sands). The orthodontic treatment in the permanent dentition was performed with fixed appliances around the age of 12-15 years followed by fixed retention at the maxillary and mandibular anterior teeth and/or a Hawley retainer in the maxilla. Lastly, in case of severe dysgnathia, combined orthodontic-surgical treatment followed by lip/nose correction was performed, if indicated.

All patient data were anonymized prior to analysis. The use of anonymous data collected during routine patient care is in accordance with Dutch law. Written consent of participants was obtained. This study has been carried out in accordance with the applicable Dutch legislation such as Medical Research involving Human Subjects Act and Medical Treatment Contracts Act concerning reviewal by an accredited research ethics committee. This has been confirmed by the Medical Ethical Committee of the Radboudumc Nijmegen, The Netherlands (File number: 2021-13046). 
143 Materials

144 Plaster models of 75 consecutive patients directly after (T0), 2 years after (T2) and five years

145 after treatment (T5) were available. The study started (T0) after orthodontic treatment had

146 finished, either after orthodontic treatment alone or after combined orthodontic surgical

147 treatment in case of severe dysgnathia. All plaster casts were scanned with a 3Shape R500 3D

148 Dental Laser scanner (3ShapeR C, Copenhagen, Denmark) using the high-resolution setting,

149 producing a spatial resolution of $0.01 \mathrm{~mm}$ as specified by the manufacturer. The occlusal

150 relationship of all models was checked against the intra-oral pictures taken at the same date.

151

152 Methods

153 The mandibular transverse dental arch dimensions were measured by one observer on the digital

154 models at T0, T2 and T5 using the open-source software MeshLab 2016 (Visual Computing Lab

155 ISTI - CNR, Pisa, Italy) (Cignoni et al., 2008). The mandibular inter-canine width was measured

156 between the left and right cusp tips, the inter first and second premolar width between the left

157 and right buccal cusp tips, and the inter-first and second molar width between the mesio-buccal

158 cusp tips (Figure 1). To determine the error of the method 20 randomly selected digital dental

159 casts were remeasured by the same observer after a one-week interval.

160 The transverse occlusal relationship was determined by applying the MHB-index (Modified

161 Huddart-Bodenham index) to evaluate arch constriction for each tooth pair. This was done on

162 plaster casts set out on tables in a quiet room by one observer. This index was first developed in

1631972 (Huddart and Bodenham, 1972) then modified in 1997 (Heidbüchel and Kuijpers-Jagtman,

164 1997). For the scoring the dentition is divided into a labial segment, and a buccal cleft and non-

165 cleft segment (Figure 2). In the labial segment the lateral incisors are not scored as they are often

166 missing or misplaced. In the buccal segments, canines, premolars, and first molars are scored.

167 Each tooth pair can receive a score from -3 to +1 (Figure 3). The total MHB score for a given

168 model is calculated as the sum of the scored teeth, with a range of the score from -30 to +10

169 (Noverraz et al, 2015). To determine the intra- and interobserver reliability 50 randomly selected

170 casts were scored twice with an interval of one week by two observers.

171

172 Statistical analysis

173 Descriptive statistics were performed for all parameters to show the distribution of data, such as

174 means, standard deviations, minimum - maximum values, mean increments between T0 and T2,

$175 \mathrm{~T} 2$ and T5, and T0 and T5. The intra-observer performance for the transverse mandibular

176 distances (continuous variables) was analyzed using three statistics. The reliability was tested

177 with the Pearson correlation coefficient. Paired sample t-tests were applied to identify systematic

178 differences between the first and second measurement. The duplicate measurement error (DME)

179 was calculated as the SD of the difference between two observations divided by $\sqrt{2}$.

180 Additionally, Bland-Altman plots were made for each variable. The duplo measurements for the

181 intra- and interobserver reliability of the individual teeth scores of the MHB Index score were

182 analyzed by weighted Kappa. Data was analyzed using paired t-tests and ANOVA. Linear

Peer] reviewing PDF | (2021:07:63491:1:0:NEW 6 Nov 2021) 
183

184

185

186

187

188

189

190

191

192

193

194

195

196

197

198

199

200

201

202

203

204

205

206

207

208

209

210

211

212

213

214

215

216

217

218

219

220

221

222

223

regression was applied to test for the effect of age at the end of treatment, sex, opening or closing space of the maxillary lateral incisor at the cleft side, mandibular premolar extraction, maxillary expansion before bone grafting, and treatment outcome (GOSLON Yardstick score) at the end of treatment. The level of significance was set at $\mathrm{P}<0.05$.

\section{Results}

\section{Sample}

The total number of patients that met the inclusion criteria was $n=82$. Data of 7 patients were missing because 3 moved elsewhere, for 3 patients all treatment records were missing, and 1 patient had no follow-up for unknown reasons. In total 75 consecutive patients were available for the study. The mean age at the end of treatment (T0) was 16.5 years (SD 2.3; range 12.9 - 22.5), two years after treatment (T2) 18.4 years (SD 2.2; range 14.6 - 22.7), and five years after treatment (T5) 20.9 years (SD 2.4; range 17.0 - 26.4). 27 patients were female (36\%) and 48 patients were male (64\%). 54 patients $(72 \%)$ had the cleft on the left side and 21 patients $(28 \%)$ on the right side. Maxillary expansion before bone grafting was done in 20 patients out of 75 (26.7\%). In 54 patients (72\%) the space for the missing maxillary lateral incisor at the cleft side was closed orthodontically, in 6 patients $(8 \%)$ the maxillary lateral incisor was present at the cleft side and in 15 patients (20\%) the space was opened. Absence of mandibular premolars was found in 19 patients $(25.3 \%)$. The GOSLON score at the end of treatment was in $36(48 \%)$ patients score 1 , in $19(25.3 \%)$ patients score 2 , in $14(18.7 \%)$ patients score 3 , and score 4 was found in $4(5.3 \%)$ patients. There were no patients with a score of 5 in this sample. Data for the GOSLON were missing of two patients.

\section{Intra- and interobserver performance}

The Pearson's correlation coefficients for the intra-observer reliability of the transverse arch measurements were high, ranging from 0.932 to 0.996 (Supplementary Table 1). The duplicate measurement error for the measurements varied from 0.19 to $0.54 \mathrm{~mm}$. The mean differences between first and second measurements were very small and showed no significant differences (paired sample t-test). This indicated that intra-observer performance was high. This was also supported by the Bland Altman plots showing a high level of agreement between the first and second measurements (Supplementary Figure 1a-c).

For the MHB score the intra-observer performance was almost perfect shown by the weighted kappa of 0.93 . The inter-observer reliability was also strong with a weighted kappa value of 0.84 . This was also supported by the Bland Altman plots showing a high level of agreement between measurements (Supplementary Figure 2a-b).

\section{Mandibular transverse dimensions}

Table 1 shows means and SD of the five mandibular transverse dimensions at the three different time points and Table 2 shows the changes over the five-year study period. The mandibular intercanine distance did not change significantly over time. The interpremolar distances 
224 diminished significantly over the 5-year study period. The first premolar distance diminished $2250.71 \pm 1.38 \mathrm{~mm}(\mathrm{p}=0.001,95 \%$ CI $0.29 \ldots 1.12)$, while the second premolar distance decreased $2260.95 \pm 1.74 \mathrm{~mm}(\mathrm{p}<0.001,95 \%$ CI $0.47 \ldots 1.43)$. The inter first molar distance increased slighty 227 over the 5-year follow-up period but this change was not statistically significant, while the inter

228

229

230

231

232

233

234

235

236

237

238

239

240

241

242

243

244

245

246

247

248

249

250

251

252

253

254

255

256

257

258

259

260

261

262

263

264 second molar distance showed an increase of $0.73 \pm 1.49 \mathrm{~mm}(\mathrm{p}=0.001,95 \%$ CI $-1.13 \ldots-0.33)$.

\section{Transverse dental arch relationships}

Table 3 shows means and SD for the MHB scores at the three different time points and Table 4 shows the changes after treatment over the five-year study period. For all arch segments - labial and cleft and non-cleft buccal segment - the MHB scores deteriorated significantly over the 5year follow-up period and during each post-treatment period. The decrease for the labial segment was small $(0.56 \pm 1.45 \mathrm{MHB}$ point, $\mathrm{p}=0.006,95 \% \mathrm{CI} 0.17 \ldots 0.96)$ during the 5 -year period, while the change for the buccal cleft segment was the largest $(2.07 \pm 2.67$ MHB point, $p<0001$, $95 \%$ CI $1.35 \ldots 2.79)$.

\section{Factors that influence changes of mandibular transverse distances}

Table 5 shows the results of the linear regression analysis for factors affecting the changes of the mandibular transverse distances for all time intervals. The deterioration of the intercanine distance two years after treatment (T0-T2) was affected by sex and GOSLON score at the end of treatment, showing that deterioration was less for girls and more when a higher GOSLON score was present at the end of treatment $\left(\mathrm{R}^{2}=0.21\right)$. The deterioration of the inter first-premolar distance was larger when the space was closed for a missing maxillary lateral $\left(\mathrm{R}^{2}=0.15\right)$. Between two and five years after treatment (T2-T5), the decrease of the mandibular inter first molar width was affected by the GOSLON score at T0 showing that the decrease was less when the GOSLON at T0 was smaller $\left(\mathrm{R}^{2}=0.14\right)$.

For the whole 5-year period (T0-T5) only maxillary expansion had a significant effect, i.e. the intercanine distance decreased more when maxillary expansion was performed during treatment, but the explained variance was low $\left(\mathrm{R}^{2}=0.13\right)$.

Yet overall, no clear picture was emerging when looking at the effect of the different factors on the changes of the distances.

\section{Factors that influence changes in the MHB Scores}

Table 6 shows the results of the linear regression analysis for contributing factors for changes of the MHB-scores. The influencing factors had the greatest effect the first two years after treatment. From T0-T2 deterioration of the labial segment was affected by mandibular premolar absence showing that the deterioration was less if the mandibular premolar was absent $\left(\mathrm{R}^{2}=\right.$ 0.17). On the buccal cleft segment, the deterioration was higher if the patient was younger at the end of treatment, space closure of a missing maxillary lateral incisor was conducted, and the GOSLON score at the end of treatment was higher $\left(\mathrm{R}^{2}=0.31\right)$. On the buccal non-cleft segment, the deterioration was affected by patient's age at the end of treatment and space closure for a missing maxillary lateral, showing that less deterioration happened if the patient was older at the 
265

266

267

268

269

270

271

272

273

274

275

276

277

278

279

280

281

282

283

284

285

286

287

288

289

290

291

292

293

294

295

296

297

298

299

300

301

302

303

304

end of treatment and no space closure was conducted for a missing maxillary lateral $\left(\mathrm{R}^{2}=0.21\right)$. The deterioration of the total arch constriction score was smaller when the patient was older at the end of treatment, no space closure was conducted for a missing maxillary lateral and the GOSLON score was smaller at the end of treatment $\left(\mathrm{R}^{2}=0.29\right)$.

Between two and five years after treatment (T2-T5), the deterioration on the buccal non-cleft segment was larger if the mandibular premolar was absent $\left(\mathrm{R}^{2}=0.15\right)$ and the total score was more deteriorated if maxillary expansion was conducted $\left(\mathrm{R}^{2}=0.20\right)$. For the total post-treatment period (T0-T5), age had an effect on the scores of the buccal cleft segment and the total arch constriction. The scores deteriorated less if the patients were older at the end of treatment $\left(\mathrm{R}^{2}=0.30\right.$ and $\mathrm{R}^{2}=0.26$, respectively).

Overall, the effect of different factors on the deterioration of interarch relationships remained unclear because all of the explained variances were low.

\section{Discussion}

The aim of this research was to determine how the mandibular dental arch develops in patients with CUCLAP from the point the treatment has finished to five years after the treatment. We considered mandibular changes because it is well-known that the maxillary arch in patients with CUCLAP is prone to changes after treatment, whereas mandibular dental arch changes or adaptation to occlusion may be expected. Only a few studies have been performed on long-term changes after treatment in adult patients with CUCLAP and we found none focusing on the transverse dental arch dimensions in the mandible. Therefore, comparison with findings from the literature is not possible as data are lacking. The null hypothesis could not be confirmed as the present study shows that the mandibular arch does change after the treatment has finished as shown by changes of the transverse dimensions and interarch relationship over the 5-year followup period.

The mandibular intercanine distance did not change significantly over time as could be expected because $85.3 \%$ of the patients $(64 / 75)$ had a mandibular canine-to-canine retainer bonded to all anterior teeth. The interpremolar distances diminished significantly over the 5-year study period with about $1 \mathrm{~mm}$. The first and second molar distance increased slightly, but this was only significant for the second molars $(0.73 \mathrm{~mm})$. The latter can be explained by uprighting of the mandibular molars buccally from a more lingually oriented crown torque which occurs with age in untreated dentitions leading to an increase of the intermolar distance (Marshall et al., 2003; Hesby et al., 2006; Yang and Chung, 2019). However, it should be noticed that changes of the intermolar distance were minor which is consistent with earlier longitudinal studies (Thilander, 2009; Heikinheimo et al., 2012; Garib et al., 2021).

In our study the interpremolar distances diminished slightly while studies on untreated subjects have shown that interpremolar distances in the mandible reached stability between 16 and 31 years of age (Thilander, 2009) and remained stable from 13 to 60 years of age (Massaro et al., 2018). These changes in the interpremolar distances might be explained as an adaptation to changes in the maxillary dental arch in CUCLAP which is subject to the ongoing constrictive 
305

306

307

308

309

310

311

312

313

314

315

316

317

318

319

320

321

322

323

324

325

326

327

328

329

330

331

332

333

334

335

336

337

338

339

340

341

342

343

344

effect of palatal scar tissue as has been shown in animal experiments in which animals were followed into adulthood (Wijdeveld et al., 1991; Kim et al., 2002; Van De Water et al., 2013).

In contrast to the lack of data on mandibular arch dimensions in patients with orofacial clefts there are a few studies that reported posttreatment changes of the occlusion and maxillary arch dimensions. Marcusson and Paulin (2004) evaluated occlusion and maxillary arch dimensions from 19 to 25 years of age with the MHB Index and found that the deteriorations were significant in all three segments. Semb, Rønning \& Åbyholm (2011) did a long-term study on antero-posterior relationship using the GOSLON Yardstick and observed that from 16 to 20 years of age in $30 \%$ of patients the GOSLON score worsened. The changes were partly due to continuous mandibular growth. A series of studies from Brazil in a convenience UCLP sample reported on short term changes (one year after treatment) primarily of the maxillary dental arch in relation to rehabilitation with a dental implant or anterior fixed bridgework (Caballero et al., 2019; Rezende Pucciarelli et al., 2020, 2021; Soares et al., 2021a,b). These studies showed that the maxillary arch was not stable until one year posttreatment regardless of the type of prosthetic rehabilitation. In our research, the MHB scores deteriorated up to 4 points for the total score which means there is an increase in the number of teeth in crossbite. The interarch relationship deteriorated and maxillary arch changes in the long term may be responsible for the increased MHB scores. Yet the mandibular arch showed only minor changes of the transverse dimensions. It means that the mandibular arch did not adapt completely to the changes in the maxillary arch dimensions because if it did the MHB scores would have remained the same.

We also studied factors which may influence the changes of the mandibular arch and the MHB score after treatment. We did not find a clear pattern for the effect of age at the end of treatment, sex, maxillary expansion before bone grafting, space closure for missing maxillary lateral, mandibular premolar absence or GOSLON score at the end of treatment, on the mandibular transverse dimensions over the 5-year follow-up period. For the interarch relationship we found that age at the end of treatment, space closure for a missing maxillary lateral incisor and GOSLON score at the end of treatment seemed to have an effect especially in the first two years after treatment. Patients who were younger at the end of treatment tended to have more changes, probably because of remaining growth. Indeed, it has been reported that patients with clefts would mature slower than non-clefts due to delayed pubertal growth (Cesur et al., 2018). Therefore, it should be taken into account that there may be a risk of post-treatment changes if we finish treatment while growth has not ceased. We also found that the posttreatment changes were less if patients had a lower GOSLON score at the end of treatment, which stands for a better sagittal jaw relationship. This is encouraging to know because it helps us to inform the patients what to expect of their treatment stability.

This study shows that changes after treatment occurred especially during the first two years after treatment. Therefore, clinicians should anticipate with their retention protocol. Bonded retainers to all anterior teeth are recommended to hold the anterior tooth positions. It is recommended to use, besides the bonded retainers, a Hawley retainer during the night to maintain the transverse dimensions after treatment. Due to the long-term effect of palatal scar

Peer] reviewing PDF | (2021:07:63491:1:0:NEW 6 Nov 2021) 
345 tissue the patient must be advised to use the Hawley retainer life-long. It was also shown that 346 patients who had a good GOSLON score at the end of treatment maintained a better transverse 347 dental arch relationship overtime. Therefore, it pays off to strive for the lowest GOSLON score 348 at the end of treatment, although this is not always possible due to the individual growth pattern 349 of the patient.

350

351

352

353

354

355

356

357

358

359

360

361

362

363

364

365

366

367

368

369

370

371

372

373

374

375

376

377

378

379

380

381

382

383

384

A common feature in patients with CUCLAP is agenesis of the maxillary lateral incisor at the cleft side. In this study we found that if the space of missing maxillary lateral was closed the interarch deterioration in the first two years after treatment was larger compared to opening the space for a lateral incisor. This may be explained by the fact that closing the space narrows the maxillary dental arch facilitating a cross bite tendency which will be reflected in deterioration of the MHB index. This raises the question whether it would be wise to open the space and replace the missing maxillary lateral (temporarily) with a Maryland bridge or dental implant. A single dental implant in the anterior region can only be placed after vertical facial growth has ceased (Kuijpers \& Loomans, 2015; Aarts et al., 2015). A noticeable infra-occlusion may also occur due to continuous vertical eruption of the adjacent teeth even though implant placement is done at mature age (Bernard et al., 2004). Furthermore, in many cases additional bone grafting is necessary before dental implant placement because bone volume loss occurs after secondary bone grafting when no tooth has erupted or placed orthodontically in the area (Stasiak, Wojtaszek-Słomińska \& Racka-Pilszak, 2019; Wermker et al., 2014). Also, peri-implant soft tissues are esthetically less pleasing in patients who did not receive additional bone augmentation as the implant cannot be placed in its optimal position due to an insufficient bone volume (Alberga et al. (2020). However, so far there are no long-term studies of dental implants in patients with clefts comparing therapies, risks, and outcome (Wermker et al., 2014; Wang et al., 2014).

There is still a lack of studies into long-term changes in patients with CUCLAP not only regarding the dental arch relationship and facial changes but also regarding other aspects of treatment outcome. The adult individual with a treated cleft deserves more attention in cleft research.

\section{Strengths and weaknesses of the study}

This study is performed in a large sample of consecutive patients observed for 5 years after treatment. There are hardly any studies about stability after treatment focusing on the mandibular arch. The loss to follow up was low (only 7 out of 82 patients - less than $10 \%$ ). The patients were treated from birth on in one center with the same protocols by two experienced surgeons which is important to notice as it has been proven that the skills of the surgeon play an important role in the final result (Shaw and Semb, 2017). However, the present study is a retrospective study and performed in one center which means the results are valid for Caucasians and for the treatment protocol which is employed in the center.

\section{Conclusions}

Peer) reviewing PDF | (2021:07:63491:1:0:NEW 6 Nov 2021) 
385

386

387

388

389

390

391

392

393

394

395

396

397

398

399

400

401

402

403

404

405

406

407

408

409

410

411

412

413

414

415

416

417

418

419

420

421

422

423

424

In patients with CUCLAP, changes occurred in the mandibular arch expressed as changes in the transverse dimensions and interarch relationship measured by the MHB Index. A younger age at the end of treatment, space closure for a missing maxillary lateral incisor and a higher GOSLON score at the end of treatment negatively influence the interarch transverse deterioration especially in the first two years after treatment. For the transverse dimensional changes in the mandibular arch such influencing factors could not be determined.

\section{Acknowledgements}

We are grateful for the contributions by Lovina Boen, DDS (Radboudume Nijmegen) and Dr. Nia Ayu Ismaniati, DDS PhD (Universitas Indonesia Jakarta) for their support in the data collection.

\section{References}

Aarts BE, Convens J, Bronkhorst EM, Kuijpers-Jagtman AM, Fudalej PS. 2015. Cessation of facial growth in subjects with short, average, and long facial types - Implications for the timing of implant placement. J Craniomaxillofac Surg 43(10):2106-2111. DOI: 10.1016/j.jcms.2015.10.013.

Alberga JM, Stellingsma K, Meijer HJA, Oostenbrink HA, Vissink A, Raghoebar GM. 2020. Dental implant placement in alveolar cleft patients: a retrospective comparative study on clinical and aesthetic outcomes. Int J Oral Maxillofac Surg 49(7):952-959. DOI: 10.1016/j.ijom.2020.02.007. Al-Gunaid T, Asahito T, Yamaki M, Hanada K, Takagi R, Ono K, Saito I. 2008. Relapse tendency in maxillary arch width in unilateral cleft lip and palate patients with different maxillary arch forms. Cleft Palate Craniofac J 45(3):278-83. DOI: 10.1597/07-053.

American Cleft Palate-Craniofacial Association. 2018. Parameters for Evaluation and Treatment of Patients with Cleft Lip/Palate or Other Craniofacial Differences.

Bernard JP, Schatz JP, Christou P, Belser U, Kiliaridis S. 2004. Long-term vertical changes of the anterior maxillary teeth adjacent to single implants in young and mature adults. A retrospective study. J Clin Periodontol 31(11):1024-8. DOI: 10.1111/j.1600-051X.2004.00574.x. Caballero JT, Rezende Pucciarelli MG, Pazmiño VFC, Curvêllo VP, Menezes M, Sforza C, Soares S. 2019. 3D comparison of dental arch stability in patients with and without cleft lip and palate after orthodontic/rehabilitative treatment. J Appl Oral Sci 27:e20180434.

DOI:10.1590/1678-7757-2018-0434

Carbullido MK, Dean RA, Kamel GN, Davis GL, Hornacek M, Segal RM, Ewing E, Lance SH, Gosman AA. 2021. Long-term treatment outcomes of primary alveolar bone grafts for alveolar clefts: A Qualitative Systematic Review. Cleft Palate Craniofac J 25:1055665621995047. DOI: $10.1177 / 1055665621995047$.

Cesur E, Altug AT, Toygar-Memikoglu U, Gumru-Celikel D, Tagrikulu B, Erbay E. 2018. Assessment of sella turcica area and skeletal maturation patterns of children with unilateral cleft lip and palate. Orthod Craniofac Res 21(2):78-83. DOI: 10.1111/ocr.12219. 
425 Cignoni P, Callieri M, Corsini M, Dellepiane M, Ganovelli F, Ranzuglia G. 2008. MeshLab: an 426 Open-Source Mesh Processing Tool. Sixth Eurographics Italian Chapter Conference. 129-136. 427 Dixon MJ, Marazita ML, Beaty TH, Murray JC. 2011. Cleft lip and palate: understanding 428 genetic and environmental influences. Nat Rev Genet 12(3):167-78. DOI: 10.1038/nrg2933. 429 Emodi O, Noy D, Hazan-Molina H, Aizenbud D, Rachmiel A. 2015. Secondary bone grafting of 430 the cleft maxilla following reverse quad-helix expansion in 103 patients. Ann Maxillofac Surg 431 5(1):32-6. DOI: 10.4103/2231-0746.161056.

432 Garib D, Miranda F, Massaro C, Lauris JRP, Yatabe MS, Janson G, McNamara JA Jr, Behrents 433 RG, Cevidanes LHS, Ruellas ACO. 2021. Three-dimensional mandibular dental changes with 434 aging. Am J Orthod Dentofacial Orthop 159(2):184-192. DOI: 10.1016/j.ajodo.2019.12.021. 435 Heikinheimo K, Nyström M, Heikinheimo T, Pirttiniemi P, Pirinen S. 2012. Dental arch width, 436 overbite, and overjet in a Finnish population with normal occlusion between the ages of 7 and 32 437 years. Eur J Orthod 34(4):418-26. DOI: 10.1093/ejo/cjr025.

438 Hesby RM, Marshall SD, Dawson DV, Southard KA, Casko JS, Franciscus RG, Southard TE. 439 2006. Transverse skeletal and dentoalveolar changes during growth. Am J Orthod Dentofacial 440 Orthop 130(6):721-31. DOI: 10.1016/j.ajodo.2005.03.026.

441 Heidbuchel KL, Kuijpers-Jagtman AM. 1997. Maxillary and mandibular dental-arch dimensions 442 and occlusion in bilateral cleft lip and palate patients from 3 to 17 years of age. Cleft Palate 443 Craniofac J 34(1):21-6. DOI: 10.1597/15451569_1997_034_0021_mamdad_2.3.co_2.

444 Huddart AG, Bodenham RS. 1972. The evaluation of arch form and occlusion in unilateral cleft 445 palate subjects. Cleft Palate J 9:194-209.

446 Kadir A, Mossey PA, Blencowe H, Moorthie S, Lawn JE, Mastroiacovo P, Modell B. 2017.

447 Systematic review and meta-analysis of the birth prevalence of orofacial clefts in low- and 448 middle-income countries. Cleft Palate Craniofac J 54(5):571-581. DOI: 10.1597/15-221.

449 Kappen IFPM, Yoder WR, Mink van der Molen AB, Breugem CC. 2018. Long-term craniofacial 450 morphology in young adults treated for a non-syndromal UCLP: A systematic review. J Plast 451 Reconstr Aesthet Surg 71(4):504-517. DOI: 10.1016/j.bjps.2017.12.007.

452 Kim T, Ishikawa H, Chu S, Handa A, Iida J, Yoshida S. 2002. Constriction of the maxillary 453 dental arch by mucoperiosteal denudation of the palate. Cleft Palate Craniofac J 39(4):425-431.

454 DOI:10.1597/1545-1569_2002_039_0425_cotmda_2.0.co_2

455 Kloukos D, Fudalej P, Sequeira-Byron P, Katsaros C. 2018. Maxillary distraction osteogenesis 456 versus orthognathic surgery for cleft lip and palate patients. Cochrane Database Syst Rev. 457 8(8):CD010403. DOI: 10.1002/14651858.CD010403.pub3.

458 Kuijpers MA, Loomans B. 2015. De gecombineerde orthodontisch restauratieve behandeling 459 [Combined orthodontic and restorative treatment]. Ned Tijdschr Tandheelkd 122(11):575-581. 460 DOI:10.5177/ntvt.2015.11.15190.

461 Marcusson A, Paulin G. 2004. Changes in occlusion and maxillary dental arch dimensions in 462 adults with treated unilateral complete cleft lip and palate: a follow-up study. Eur J Orthod 463 26(4):385-90. DOI: 10.1093/ejo/26.4.385. 
464 Marshall S, Dawson D, Southard KA, Lee AN, Casko JS, Southard TE. 2003. Transverse molar

465

466

467

468

469

470

471

472

473

474

475

476

477

478

479

480

481

482

483

484

485

486

487

488

489

490

491

492

493

494

495

496

497

498

499

500

501

502

movements during growth. Am J Orthod Dentofacial Orthop 124(6):615-24. DOI:

10.1016/s0889-5406(03)00630-9.

Martelli DR, Machado RA, Swerts MS, Rodrigues LA, Aquino SN, Martelli Júnior H. 2012. Non syndromic cleft lip and palate: relationship between sex and clinical extension. Braz J Otorhinolaryngol 78(5):116-20. English, Portuguese. DOI: 10.5935/1808-8694.20120018. Massaro C, Miranda F, Janson G, Rodrigues de Almeida R, Pinzan A, Martins DR, Garib D. 2018. Maturational changes of the normal occlusion: A 40-year follow-up. Am J Orthod Dentofacial Orthop 154(2):188-200. DOI: 10.1016/j.ajodo.2017.10.028.

Mossey PA, Modell B. 2012. Epidemiology of oral clefts 2012: an international perspective. Front Oral Biol 16:1-18. DOI: 10.1159/000337464.

Noverraz RL, Disse MA, Ongkosuwito EM, Kuijpers-Jagtman AM, Prahl C. 2015. Transverse dental arch relationship at 9 and 12 years in children with unilateral cleft lip and palate treated with infant orthopedics: a randomized clinical trial (DUTCHCLEFT). Clin Oral Investig 19(9):2255-65. DOI: 10.1007/s00784-015-1451-2.

Oosterkamp BC, Dijkstra PU, Remmelink HJ, van Oort RP, Sandham A. 2010. Orthodontic space closure versus prosthetic replacement of missing upper lateral incisors in patients with bilateral cleft lip and palate. Cleft Palate Craniofac J 47(6):591-6. DOI: 10.1597/09-092.

Rezende Pucciarelli MG, de Lima Toyoshima GH, Marchini Oliveira T, Marques Honório H, Sforza C, Soares S. 2020. Assessment of dental arch stability after orthodontic treatment and oral rehabilitation in complete unilateral cleft lip and palate and non-clefts patients using 3D stereophotogrammetry. BMC oral health 20(1):154. DOI:10.1186/s12903-020-01143-1 Pucciarelli MGR, Toyoshima GH, Cardoso JF, de Oliveira TM, Neppelenbroek KH, Soares S. Arch asymmetry in patients with cleft lip and palate after rehabilitation treatment using stereophotogrammetry. J Craniofac Surg. 2021;32(5):e501-e504.

doi:10.1097/SCS.0000000000007460

Semb G, Rønning E, Åbyholm F. 2011. Twenty-year follow-up of 50 consecutive patients born with unilateral complete cleft lip and palate treated by the Oslo Cleft Team, Norway. Semin Orthod 17:207-224. DOI:10.1053/j.sodo.2011.02.005.

Shaw W, Semb G. 2017. The Scandcleft randomised trials of primary surgery for unilateral cleft lip and palate: 11. What next? J Plast Surg Hand Surg 51(1):88-93. DOI:

10.1080/2000656X.2016.1254644.

Soares S, Rezende Pucciarelli MG, de Lima Toyoshima GH, Marchini Oliveira T. 2021a. Arch asymmetry in patients with cleft lip and palate after rehabilitation treatment using stereophotogrammetry. J Craniofac Surg 2021;32(5):e501-e504. DOI:10.1097/SCS.0000000000007460 Soares S, Rezende Pucciarelli MG, de Lima Toyoshima GH, Marchini Oliveira T. 2021b. Stereophotogrammetry to evaluate young adults with and without cleft lip and palate after orthodontic and restorative treatment. J Prosthet Dent Feb 12:S0022-3913(20)30733-2. DOI: 10.1016/j.prosdent.2020.10.025. Epub ahead of print.

Peer) reviewing PDF | (2021:07:63491:1:0:NEW 6 Nov 2021) 
503 Stasiak M, Wojtaszek-Słomińska A, Racka-Pilszak B. 2019. Current methods for secondary 504 alveolar bone grafting assessment in cleft lip and palate patients - A systematic review. $J$ 505 Craniomaxillofac Surg 47(4):578-585. DOI: 10.1016/j.jcms.2019.01.013.

506 Thilander B. 2009. Dentoalveolar development in subjects with normal occlusion. A longitudinal 507 study between the ages of 5 and 31 years. Eur J Orthod 31(2):109-20. DOI: 10.1093/ejo/cjn124. 508 Wang F, Wu Y, Zou D, Wang G, Kaigler D. 2014. Clinical outcomes of dental implant therapy 509 in alveolar cleft patients: a systematic review. Int J Oral Maxillofac Implants 29(5):1098-105. 510 DOI: $10.11607 /$ jomi.3585.

511 Van De Water L, Varney S, Tomasek JJ. Mechanoregulation of the myofibroblast in wound 512 contraction, scarring, and fibrosis: Opportunities for new therapeutic intervention. 2013. Adv 513 Wound Care (New Rochelle) 2(4):122-141. DOI:10.1089/wound.2012.0393

514 Wermker K, Jung S, Joos U, Kleinheinz J. 2014. Dental implants in cleft lip, alveolus, and palate 515 patients: a systematic review. Int J Oral Maxillofac Implants Mar-29(2):384-90. DOI:

$516 \quad 10.11607 /$ jomi.3303.

517 Wijdeveld MG, Maltha JC, Grupping EM, De Jonge J, Kuijpers-Jagtman AM. 1991. A

518 histological study of tissue response to simulated cleft palate surgery at different ages in beagle 519 dogs. Arch Oral Biol 36(11):837-43. DOI: 10.1016/0003-9969(91)90033-q.

520 Yang B, Chung $\mathrm{CH}$. Buccolingual inclination of molars in untreated children and adults: A cone 521 beam computed tomography study. 2019. Angle Orthod 89(1):87-92. DOI: 10.2319/010418-6.1

522 Yılmaz HN, Özbilen EÖ, Üstün T. 2019. The Prevalence of Cleft Lip and Palate Patients: A

523 Single-Center Experience for 17 Years. Turk J Orthod 32(3):139-144. DOI:

524 10.5152/TurkJOrthod.2019.18094.

525

526

527

\section{FIGURE LEGENDS}

528

529

530

\section{Figure 1}

531

532

533

534

535

536

537

538

539
The mandibular transverse dental arch dimensions

\section{Figure 2}

The segments of the maxillary arch on which the Modified Huddart-Bodenham score for interarch relationship is determined

\section{Figure 3}

The Modified Huddart-Bodenham scoring of teeth pairs to determine the interarch relationship 


\section{Table $\mathbf{1}$ (on next page)}

Mandibular intercanine, inter first and second premolar, and inter first and second molar distances directly after treatment, two and five years after treatment (in $\mathrm{mm}$ ).

Mean and standard deviation (SD) for mandibular intercanine, inter first-premolar, inter second-premolar, inter first-molar, and inter second-molar distances after treatment (TO), two years after treatment (T1), and five years after treatment (T2) (mean, SD in mm). 
1 Table 1 Mean and standard deviation (SD) for mandibular intercanine, inter first-premolar, inter 2 second-premolar, inter first-molar, inter second-molar distances after treatment (TO), two years 3 after treatment (T1), and five years after treatment (T2) (mean, SD in mm). 4

\begin{tabular}{|c|c|c|c|c|c|c|}
\hline $\begin{array}{l}\text { Mandibular } \\
\text { Distances }\end{array}$ & $\begin{array}{l}\text { Time } \\
\text { Point }\end{array}$ & $\mathbf{N}$ & Mean & SD & Minimum & Maximum \\
\hline \multirow[t]{3}{*}{$3-3$} & T0 & 75 & 26.18 & 1.93 & 21.21 & 30.34 \\
\hline & $\mathrm{T} 2$ & 65 & 26.17 & 2.16 & 21.02 & 30.78 \\
\hline & T5 & 57 & 26.16 & 2.00 & 21.30 & 30.41 \\
\hline \multirow[t]{3}{*}{$4-4$} & TO & 58 & 33.20 & 2.09 & 28.49 & 37.93 \\
\hline & $\mathrm{T} 2$ & 50 & 32.58 & 2.27 & 27.79 & 38.07 \\
\hline & T5 & 44 & 32.29 & 2.30 & 27.61 & 37.78 \\
\hline \multirow[t]{3}{*}{$5-5$} & T0 & 70 & 37.77 & 2.97 & 31.16 & 43.96 \\
\hline & $\mathrm{T} 2$ & 63 & 36.76 & 3.09 & 30.36 & 43.49 \\
\hline & T5 & 53 & 36.82 & 3.16 & 30.11 & 43.44 \\
\hline \multirow[t]{3}{*}{$6-6$} & T0 & 75 & 41.90 & 3.21 & 36.49 & 49.77 \\
\hline & $\mathrm{T} 2$ & 66 & 42.22 & 3.45 & 36.06 & 51.07 \\
\hline & $\mathrm{T} 5$ & 56 & 42.15 & 3.30 & 35.34 & 51.01 \\
\hline \multirow[t]{3}{*}{$7-7$} & T0 & 73 & 48.56 & 3.36 & 40.29 & 58.02 \\
\hline & $\mathrm{T} 2$ & 64 & 49.05 & 3.46 & 39.67 & 58.87 \\
\hline & T5 & 55 & 48.99 & 3.76 & 39.32 & 57.88 \\
\hline
\end{tabular}

5

6

7 


\section{Table 2 (on next page)}

Changes of the mandibular intercanine, first and second premolar, and first and second molar distances over the five-year post-treatment period (in $\mathrm{mm}$ ).

Changes of the mandibular intercanine (3-3), inter first-premolar (4-4), inter second premolar (5-5), first molar (6-6), and second molar (7-7) distances during the first two years after treatment (T0-T2), from two to five years after treatment (T2-T5), and for the whole five-year period after treatment (T0-T5) (mean, SD in $\mathrm{mm}$ ) (paired t-test, $\mathrm{p}<0.05$ ) 
1 Table 21 Changes of the mandibular intercanine (3-3), inter first-premolar (4-4), inter second 2 premolar (5-5), first molar (6-6), and second molar (7-7) distances during the first two years after 3 treatment (TO-T2), from two to five years after treatment (T2-T5), and for the whole five-year 4 period after treatment (TO-T5) (mean, SD in $\mathrm{mm}$ ) (paired t-test, $\mathrm{p}<0.05$ ) 5

\begin{tabular}{|c|c|c|c|c|c|c|c|c|c|}
\hline \multirow{2}{*}{$\begin{array}{l}\text { Mandibular } \\
\text { Distance }\end{array}$} & \multirow{2}{*}{$\begin{array}{l}\text { Time } \\
\text { Point }\end{array}$} & \multirow[t]{2}{*}{$\mathbf{N}$} & \multirow[t]{2}{*}{ Mean } & \multirow[t]{2}{*}{ SD } & \multirow[t]{2}{*}{ Minimum } & \multirow[t]{2}{*}{ Maximum } & \multicolumn{2}{|c|}{$\begin{array}{c}\text { 95\% Confidence } \\
\text { Interval }\end{array}$} & \multirow[t]{2}{*}{$p$-value } \\
\hline & & & & & & & Lower & Upper & \\
\hline \multirow[t]{3}{*}{$3-3$} & $\mathrm{~T} 0-\mathrm{T} 2$ & 65 & 0.03 & 0.61 & -0.99 & 3.27 & -0.12 & 0.18 & 0.698 \\
\hline & $\mathrm{T} 2-\mathrm{T} 5$ & 50 & 0.05 & 0.44 & -0.82 & 1.15 & -0.07 & 0.18 & 0.388 \\
\hline & T0 - T5 & 57 & 0.07 & 0.72 & -1.12 & 3.61 & -0.12 & 0.26 & 0.444 \\
\hline \multirow[t]{3}{*}{$4-4$} & T0 - T2 & 49 & 0.65 & 1.09 & -2.03 & 3.38 & 0.34 & 0.96 & $<0.001$ \\
\hline & $\mathrm{T} 2-\mathrm{T} 5$ & 38 & 0.22 & 0.49 & -1.14 & 1.54 & 0.06 & 0.38 & 0.007 \\
\hline & T0 - T5 & 44 & 0.71 & 1.38 & -2.12 & 4.61 & 0.29 & 1.12 & 0.001 \\
\hline \multirow[t]{3}{*}{$5-5$} & $\mathrm{~T} 0-\mathrm{T} 2$ & 63 & 0.92 & 1.37 & -2.30 & 4.29 & 0.58 & 1.27 & $<0.001$ \\
\hline & $\mathrm{T} 2-\mathrm{T} 5$ & 48 & 0.23 & 0.67 & -1.76 & 1.66 & 0.04 & 0.42 & 0.021 \\
\hline & T0 - T5 & 52 & 0.95 & 1.74 & -3.70 & 4.70 & 0.47 & 1.43 & $<0.001$ \\
\hline \multirow[t]{3}{*}{$6-6$} & $\mathrm{~T} 0-\mathrm{T} 2$ & 66 & -0.17 & 1.18 & -4.19 & 2.66 & -0.46 & 0.12 & 0.247 \\
\hline & $\mathrm{T} 2-\mathrm{T} 5$ & 50 & 0.05 & 0.62 & -1.21 & 1.81 & -0.13 & 0.22 & 0.597 \\
\hline & T0 - T5 & 56 & -0.43 & 1.72 & -5.34 & 2.71 & -0.89 & 0.03 & 0.064 \\
\hline \multirow[t]{3}{*}{$7-7$} & $\mathrm{~T} 0$ - T2 & 64 & -0.35 & 1.15 & -3.50 & 3.19 & -0.63 & -0.06 & 0.017 \\
\hline & $\mathrm{T} 2-\mathrm{T} 5$ & 48 & -0.20 & 1.06 & -3.22 & 3.04 & -0.51 & 0.10 & 0.188 \\
\hline & T0 - T5 & 54 & -0.73 & 1.49 & -4.13 & 2.40 & -1.13 & -0.33 & 0.001 \\
\hline
\end{tabular}

6 


\section{Table 3 (on next page)}

Modified Huddart-Bodenham scores for the labial, cleft and non-cleft buccal segments and total arch constriction score directly after treatment, two and five years after treatment (in points).

Mean and standard deviation (SD) for the Modified Huddart-Bodenham scores for the labial, cleft, and non-cleft segments and total arch constriction score at the end of treatment (T0), two years after treatment (T2), and five years after treatment (T5) (in points). 
1 Table 3 Mean and standard deviation (SD) for the Modified Huddart-Bodenham scores for the 2 labial, cleft, and non-cleft segments and total arch constriction score at the end of treatment 3 (T0), two years after treatment (T2), and five years after treatment (T5) (in points). 4

\begin{tabular}{lcccccc}
\hline Segment & $\begin{array}{c}\text { Time } \\
\text { Point }\end{array}$ & N & Mean & SD & Minimum & Maximum \\
\hline Labial & T0 & 73 & 0.49 & 1.92 & -6 & 2 \\
& T2 & 64 & 0.33 & 1.73 & -6 & 2 \\
\multirow{5}{*}{ Cleft } & T5 & 56 & 0.09 & 1.74 & -6 & 2 \\
& T0 & 73 & -0.74 & 2.47 & -8 & 4 \\
& T2 & 64 & -1.95 & 2.88 & -11 & 4 \\
Non-cleft & T5 & 56 & -2.54 & 2.88 & -11 & 2 \\
& T0 & 73 & 0.56 & 1.99 & -6 & 4 \\
\multirow{4}{*}{ Total arch } & T2 & 64 & -0.33 & 1.70 & -7 & 3 \\
& T5 & 56 & -0.70 & 1.95 & -10 & 2 \\
& T0 & 73 & 0.32 & 4.92 & -17 & 8 \\
& T2 & 64 & -1.95 & 4.86 & -17 & 6 \\
\hline
\end{tabular}




\section{Table 4 (on next page)}

Deterioration of the Modified Huddart-Bodenham scores for the labial, cleft and noncleft buccal segments and total arch constriction over the five-year post-treatment period (in points).

Deterioration of the Modified Huddart-Bodenham scores for the labial, cleft and non-cleft buccal segments and total arch constriction during the first two years after treatment (T0T2), from two to five years after treatment (T2-T5), for the whole five-year period after treatment (T0-T5) (mean, SD, in points) (paired t-test, $p<0.05$ ) 
1 Table 42 Deterioration of the Modified Huddart-Bodenham scores for the labial, cleft and non-

2 cleft buccal segments and total arch constriction during the first two years after treatment (TO-

3 T2), from two to five years after treatment (T2-T5), for the whole five-year period after treatment

4 (T0-T5) (mean, SD, in points) (paired t-test, $p<0.05)$

5

\begin{tabular}{|c|c|c|c|c|c|c|c|c|c|}
\hline \multirow[t]{2}{*}{ Segment } & \multirow{2}{*}{$\begin{array}{l}\text { Time } \\
\text { Point }\end{array}$} & \multirow[t]{2}{*}{$\mathbf{N}$} & \multirow[t]{2}{*}{ Mean } & \multirow[t]{2}{*}{ SD } & \multirow[t]{2}{*}{ Minimum } & \multicolumn{3}{|c|}{$\begin{array}{c}\text { 95\% Confidence } \\
\text { Interval }\end{array}$} & \multirow[t]{2}{*}{ p-value } \\
\hline & & & & & & & Lower & Upper & \\
\hline \multirow[t]{3}{*}{ Labial } & $\mathrm{T} 0-\mathrm{T} 2$ & 63 & 0.37 & 1.11 & -2 & 4 & 0.09 & 0.65 & 0.011 \\
\hline & $\mathrm{T} 2-\mathrm{T} 5$ & 51 & 0.24 & 0.74 & -2 & 3 & 0.03 & 0.44 & 0.027 \\
\hline & T0 - T5 & 55 & 0.56 & 1.45 & -4 & 4 & 0.17 & 0.96 & 0.006 \\
\hline \multirow[t]{3}{*}{ Cleft } & $\mathrm{T} 0-\mathrm{T} 2$ & 63 & 1.62 & 2.23 & -3 & 9 & 1.06 & 2.18 & $<0.001$ \\
\hline & $\mathrm{T} 2-\mathrm{T} 5$ & 51 & 0.47 & 1.10 & -1 & 4 & 0.16 & 0.78 & 0.004 \\
\hline & T0 - T5 & 55 & 2.07 & 2.67 & -2 & 10 & 1.35 & 2.79 & $<0.001$ \\
\hline \multirow[t]{3}{*}{ Non-cleft } & T0 - T2 & 63 & 0.94 & 1.51 & -2 & 4 & 0.56 & 1.32 & $<0.001$ \\
\hline & $\mathrm{T} 2-\mathrm{T} 5$ & 51 & 0.47 & 0.86 & -1 & 3 & 0.23 & 0.71 & $<0.001$ \\
\hline & T0 - T5 & 55 & 1.38 & 1.67 & -3 & 6 & 0.93 & 1.83 & $<0.001$ \\
\hline \multirow[t]{3}{*}{ Total } & $\mathrm{T} 0-\mathrm{T} 2$ & 63 & 2.92 & 3.48 & -2 & 13 & 2.04 & 3.80 & $<0.001$ \\
\hline & $\mathrm{T} 2-\mathrm{T} 5$ & 51 & 1.18 & 1.83 & -2 & 7 & 0.66 & 1.69 & $<0.001$ \\
\hline & T0 - T5 & 55 & 4.02 & 4.38 & -9 & 17 & 2.83 & 5.20 & $<0.001$ \\
\hline
\end{tabular}

6

7

8 


\section{Table 5 (on next page)}

Influencing factors on decreases of the mandibular intercanine, inter first and second premolar, and inter first and second molar distances over the five-year post-treatment period.

Influencing factors (age, sex, maxillary expansion before bone grafting, space closure of the missing maxillary lateral incisor, absence of mandibular premolars, and GOSLON score after treatment (TO) on decreases of mandibular intercanine (D33), inter first-premolar (D44), inter second-premolar (D55), inter first-molar (D66), inter second-molar (D77) distances during the first two years after treatment (T0-T2), from two to five years after treatment (T2-T5), and for the whole five-year period after treatment(T0-T5) (Linear regression test) 
TABLE $\underline{5} 3$ Influencing factors (age, sex, maxillary expansion before bone grafting, space closure of the 2 missing maxillaryupper lateral incisor, absence of mandibulartower premolars, and GOSLON score after 3 treatment (TO) on decreases of mandibular intercanine (D33), inter first-premolar (D44), inter second4 premolar (D55), inter first-molar (D66), inter second-molar (D77) distances during the first two years after 5 treatment (T0-T2), from two to five years after treatment (T2-T5), and for the whole five-year period after 6 treatment(TO-T5) (Linear regression test)

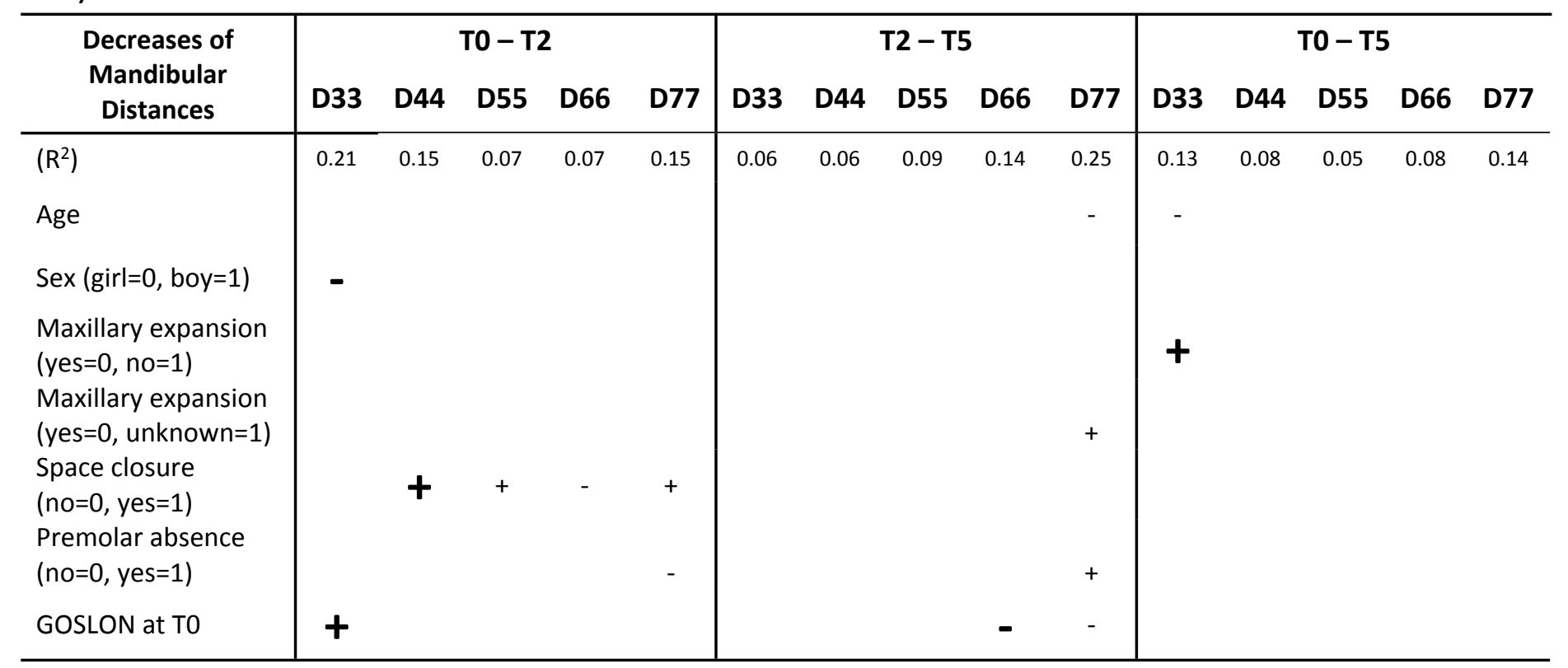

8

$9+: 0.05 \leq p \leq 0.2$ with positive effect; $+: p<0.05$ with positive effect;

$10-: 0.05 \leq p \leq 0.2$ with negative effect; $-: p<0.05$ with negative effect 


\section{Table 6(on next page)}

Influencing factors on the Modified Huddart-Bodenham scores of the labial, buccal cleft and non-cleft segment, and total arch constriction over the five-year period posttreatment.

Influencing factors (age, sex, maxillary expansion before bone grafting, space closure of missing maxillary lateral incisor, absence of mandibular premolars, GOSLON score after treatment (TO) on the Modified Huddart-Bodenham scores of the labial (Lab), buccal cleft segment (CS) and non-cleft segment (NCS), and total arch constriction (Total) during the first two years after treatment (T0-T2), from two to five years after treatment (T2-T5), and for the whole five-year period after treatment (T0-T5) (Linear regression test) 
1 TABLE 64 Influencing factors (age, sex, maxillary expansion before bone grafting, space closure of missing 2 maxillary upper-lateral incisor, absence of mandibular tower-premolars, GOSLON score after treatment 3 (TO) on the Modified Huddart-Bodenham scores of labial (Lab), buccal cleft segment (CS) and non-cleft 4 segment (NCS), and total arch constriction (Total) during the first two years after treatment (T0-T2), from 5 two to five years after treatment (T2-T5), and for the whole five-year period after treatment (T0-T5)

6 (Linear regression test)

7

\begin{tabular}{|c|c|c|c|c|c|c|c|c|c|c|c|c|}
\hline \multirow{2}{*}{$\begin{array}{l}\text { Deterioration of } \\
\text { MHB Scores }\end{array}$} & \multicolumn{4}{|c|}{$\mathrm{T} 0-\mathrm{T} 2$} & \multicolumn{4}{|c|}{$\mathrm{T} 2-\mathrm{T} 5$} & \multicolumn{4}{|c|}{ TO - T5 } \\
\hline & Lab & CS & NCS & Total & Lab & CS & NCS & Total & Lab & CS & NCS & Total \\
\hline$\left(R^{2}\right)$ & 0.17 & 0.31 & 0.21 & 0.29 & 0.10 & 0.13 & 0.15 & 0.20 & 0.12 & 0.30 & 0.21 & 0.26 \\
\hline Age & & - & - & - & & & & & & - & - & - \\
\hline Sex (girl=0, boy 1 ) & & & & & & & & & & & & \\
\hline $\begin{array}{l}\text { Maxillary expansion } \\
\text { (yes }=0, \mathrm{no}=1)\end{array}$ & - & & & & & & - & - & - & & - & - \\
\hline $\begin{array}{l}\text { Maxillary expansion } \\
(\text { yes }=0 \text {, unknown }=1 \text { ) }\end{array}$ & & & & & - & & & & & & & \\
\hline $\begin{array}{l}\text { Space closure } \\
(\text { no }=0, \text { yes }=1)\end{array}$ & & + & + & + & & & & & & + & + & + \\
\hline $\begin{array}{l}\text { Premolar absence } \\
(\text { no }=0 \text {, yes }=1)\end{array}$ & - & & & & & + & + & + & & + & & \\
\hline GOSLON at TO & & + & & + & & & & & & + & + & + \\
\hline
\end{tabular}

$+: 0.05 \leq p \leq 0.2$ with positive effect; $+: p<0.05$ with positive effect;

9

$-: 0.05 \leq p \leq 0.2$ with negative effect; $-: p<0.05$ with negative effect 
Figure 1

Mandibular transverse dental arch dimensions

Measurements of the mandibular transverse dental arch dimensions

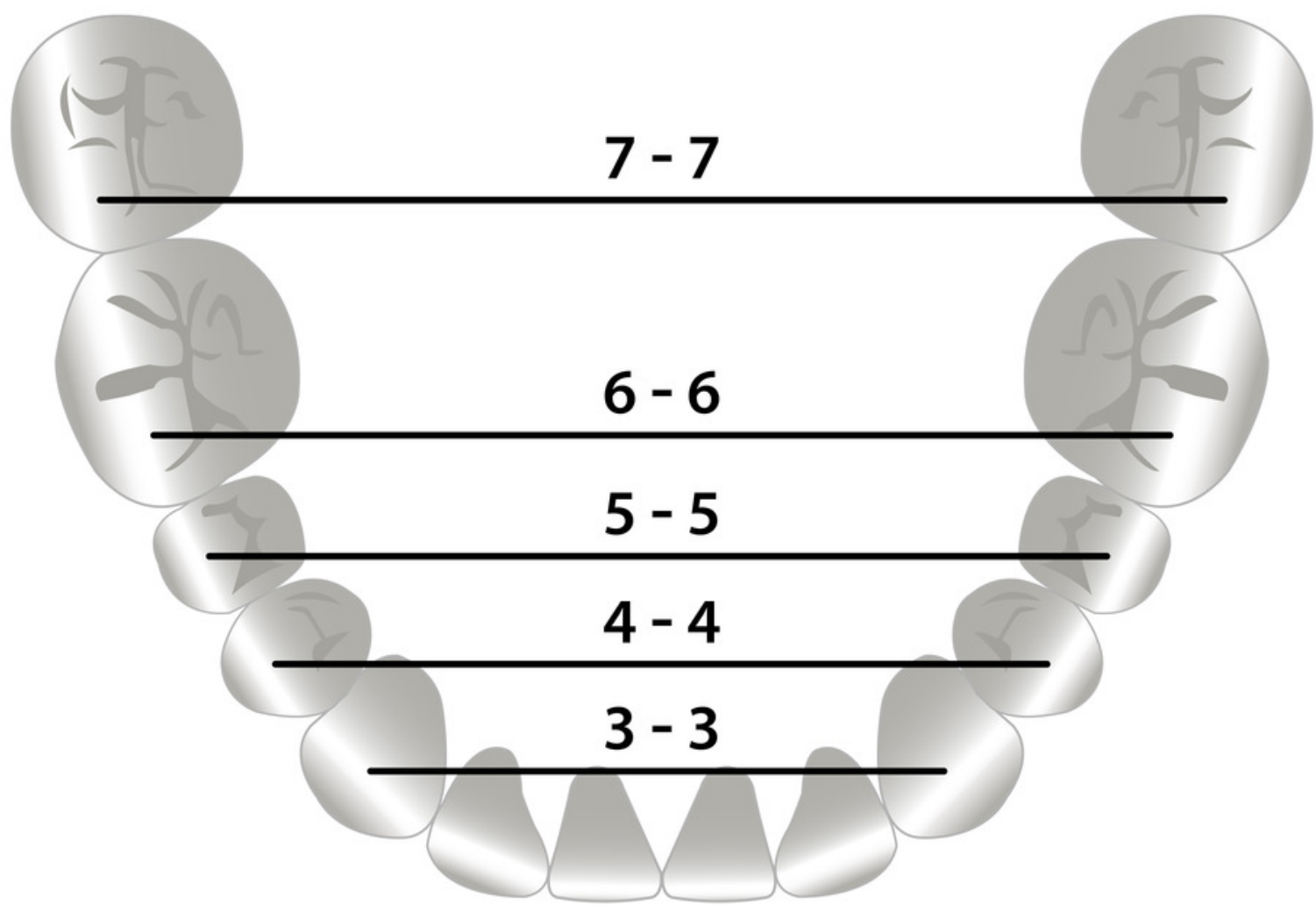


Figure 2

Maxillary arch segments used for the Modified Huddart-Bodenham scoring

The segments of the maxillary arch on which the Modified Huddart-Bodenham score for interarch relationship is determined

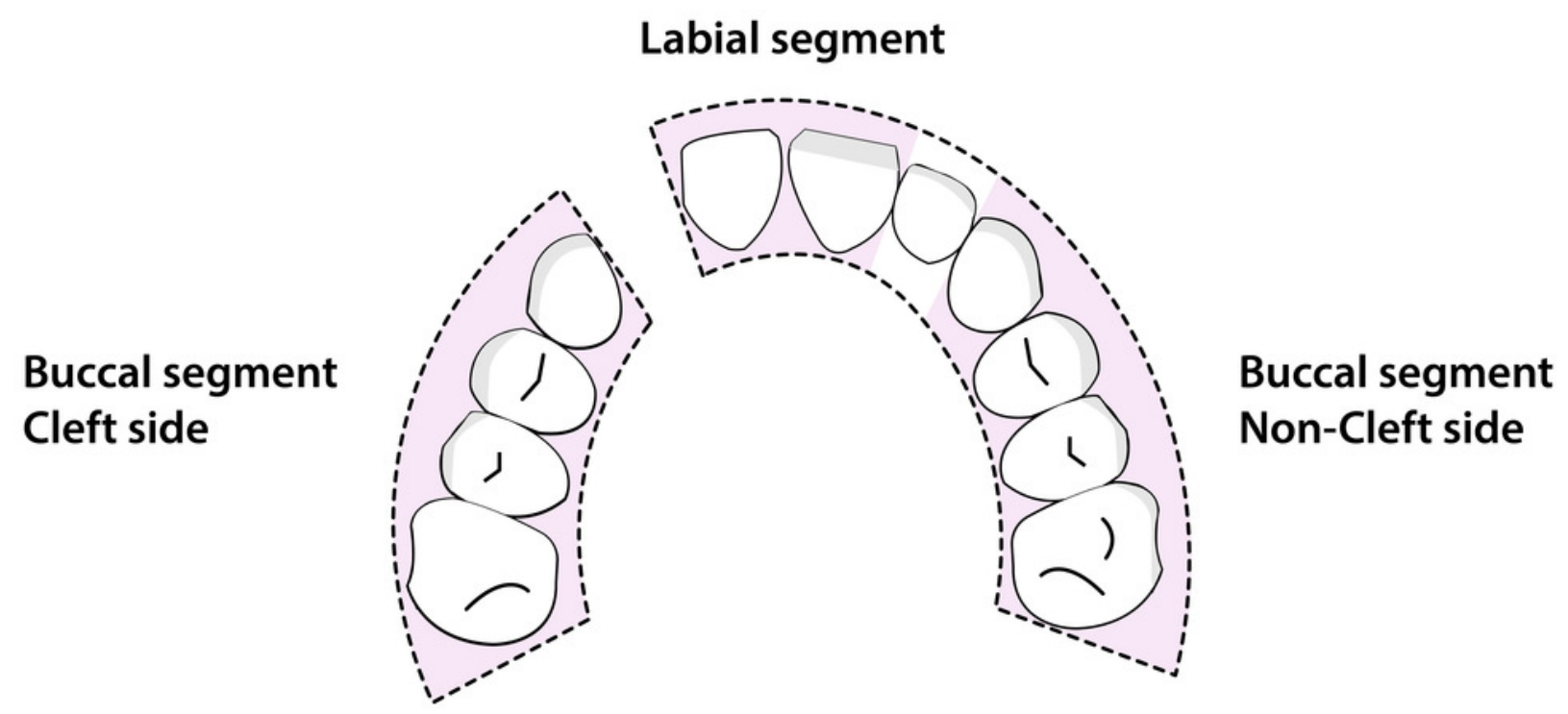


Figure 3

The Modified Huddart-Bodenham scoring

The Modified Huddart-Bodenham scoring of teeth pairs to determine the interarch relationship 


\section{Incisor scoring}

Palatal side

Palatal side

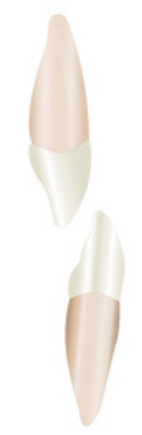

$-3$

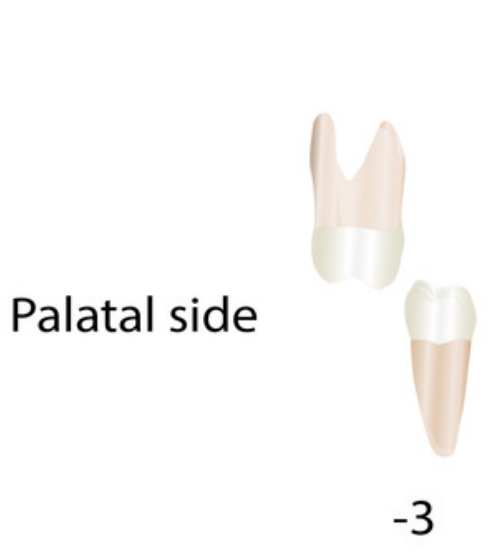

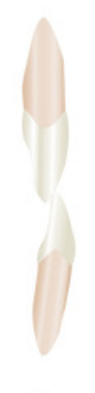

$-2$

\section{Canine scoring}
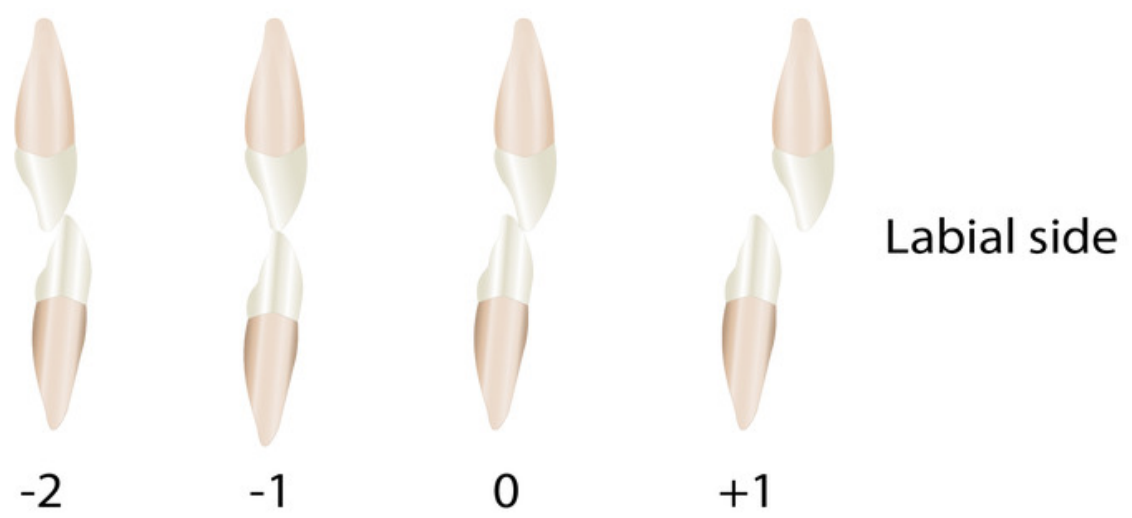

Molar scoring
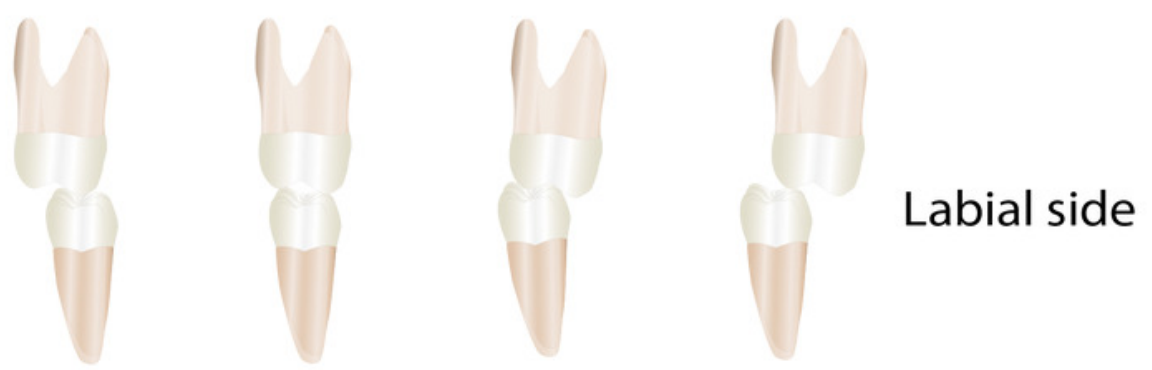

$-2$
Labial side

$+1$ 